\title{
IMPLEMENTACIÓN DE UN DATA CENTER ACADÉMICO VIRTUALIZADO
}

\author{
Manuel Yrigoyen Quintanilla \\ Carlos Martín Torres Paredes \\ Escuela de Ingeniería de la Universidad de Lima. Lima, Perú
}

Recibido: 3 de julio de 2015 / Aprobado: 17 de julio de 2015

\begin{abstract}
Resumen
El presente artículo se basa en la investigación llevada a cabo por los autores durante los años 2011 y 2012, en el marco de la ejecución de proyectos de investigación auspiciados por el Instituto de Investigación Científica de la Universidad de Lima (IDIC). En la referida investigación, se analizó la evolución tecnológica de los data center y se propuso una arquitectura que contemple los aspectos tecnológicos y de gestión de un data center virtualizado (DCV), con la finalidad de proporcionar servicios para el desarrollo de laboratorios en el ámbito académico universitario. Los hallazgos de este trabajo se aplicaron durante el proceso de planeamiento, especificación e implantación del Laboratorio de Data Center de la Carrera de Ingeniería de Sistemas de la Universidad de Lima, como parte del proceso de la implementación de su nuevo Plan de Estudios.
\end{abstract}

Palabras clave: cloud computing / infraestructura como servicios / virtualización / data center I gestión de servicios de TI

\section{Implementation of a virtualized academic data center}

\section{Summary}

This article is based on research conducted by the authors between 2011 and 2012, in the framework of the implementation of research projects, sponsored by the Scientific Research Institute of the University of Lima (IDIC). In such research, the technological evolution of the Datacenter was analyzed, and the creation of the technology and management aspects of a Virtualized Datacenter (DCV) were proposed, in order to provide services for the development of laboratories in the academic university field. The findings of this research were applied in the planning process of the "Laboratory Datacenter" of the Systems Engineering Career at the University of Lima, which was used in implementing the new syllabus.

Key words: cloud computing / infrastructure as services / virtualization / data center / IT service management 


\section{Introducción}

El data center (DC) es una de las partes más importantes de la infraestructura de las tecnologías de la información (TI) de una organización. Se compone de servidores, sistemas operativos, bases de datos, redes y de los sistemas de información de la organización, entre otros, y se aloja en un ambiente especialmente diseñado de acuerdo a los requerimientos específicos. Los data center han evolucionado de manera continua, especialmente en los últimos años, debido al desarrollo de nuevas tecnologías que reducen sus costos de adquisición (CAPEX) y operación (OPEX), simplificando, al mismo tiempo, su complejidad.

En 2012, el centro de datos continuará su transformación desde una infraestructura de TI tradicional, virtualizada, consolidada y centralizada, hacia una nube interna orientada a servicio y eficiente económicamente. El nuevo centro de datos permitirá a los usuarios internos consumir las TI como un servicio, albergar aplicaciones y datos críticos, y aumentar la capacidad a través de nubes externas. (Villarrubia, 2012)

Lo manifestado en el texto citado muestra la evolución de los data center, en la que se destaca el uso del cloud computing.

\section{El nuevo plan de estudios}

A fines de 2010, la Universidad de Lima determinó el nuevo perfil del ingeniero de sistemas formado en dicha casa de estudios. Esta descripción señala que:

El egresado (...) está preparado para afrontar los retos y las exigencias de las organizaciones, gracias a su capacidad para integrar los procesos de negocios y de proponer, desarrollar, implementar y gestionar soluciones basadas en tecnologías de información que se aplican a las mismas, permitiéndoles alcanzar sus objetivos estratégicos de una manera efectiva. (Universidad de Lima, 2012)

En la figura 1 se muestra la malla curricular de la Carrera de Ingeniería de Sistemas, que entró en vigencia en 2012. Las asignaturas se estructuraron en cuatro áreas académicas: una de Ciencias Básicas y las otras tres referidas a los aspectos de TI, conformadas por las áreas de Sistemas de Gestión, Ingeniería de Software e Infraestructura de TI.

A continuación se presentan algunas de las asignaturas definidas para las tres últimas áreas:

a) Área de Sistemas de Gestión: Sistemas de Soporte Empresarial I y II, Gestión de Procesos de Negocios, Análisis de Negocios con TI, Arquitectura Empresarial, Sistemas de Inteligencia Empresarial. 


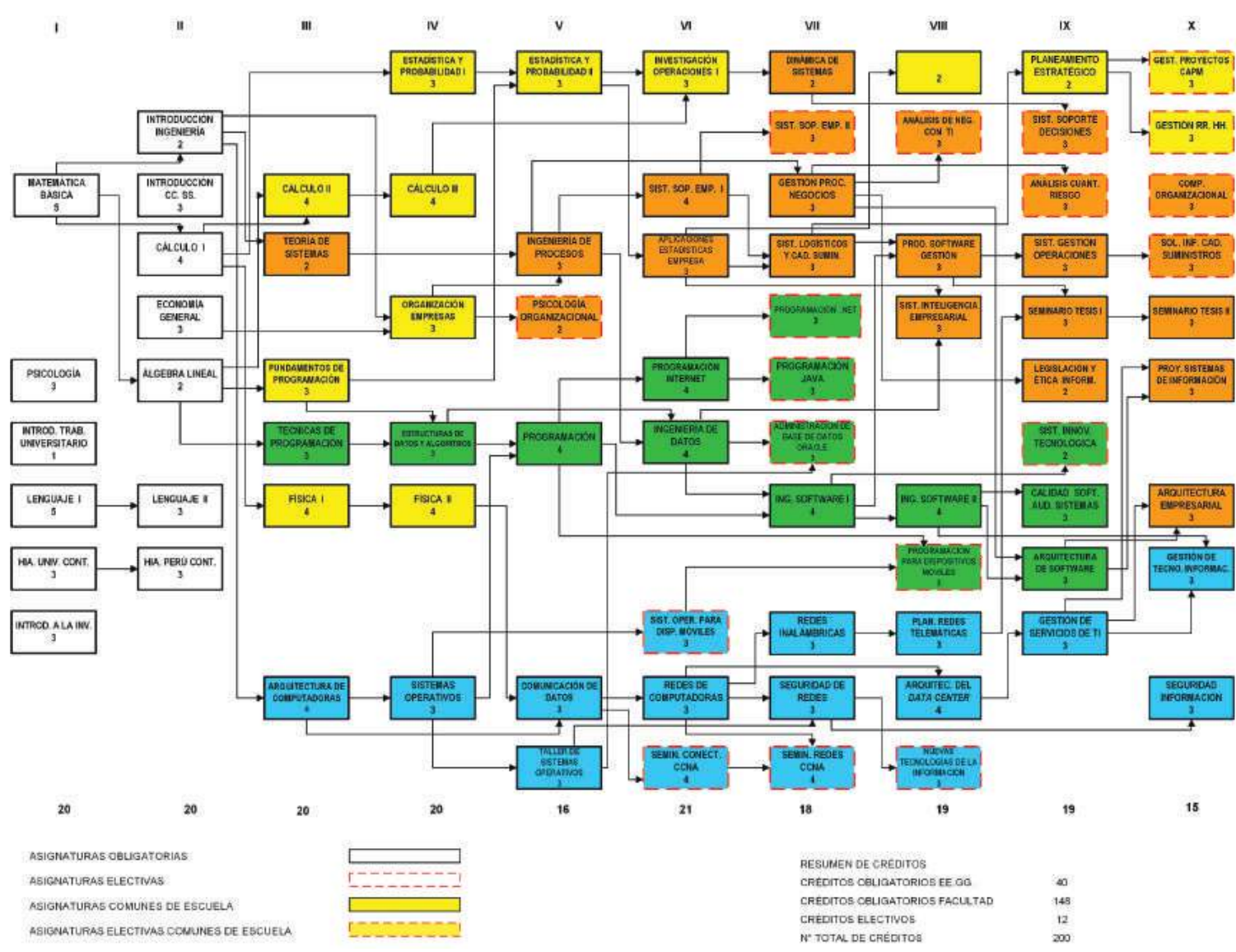

Figura 1. Malla curricular de la Carrera de Ingeniería de Sistemas 2012-1

Fuente: Carrera de Ingeniería de Sistemas - Universidad de Lima

b) Área de Ingeniería de Software: Programación para Dispositivos Móviles, Sistemas e Innovación Tecnológica, Calidad de Software y Auditoría de Sistemas, Arquitectura de Software.

c) Área de Infraestructura de TI: Sistemas Operativos para Dispositivos Móviles, Redes Inalámbricas, Seguridad de Redes, Arquitectura del Data Center, Gestión de Servicios de TI.

Otro aspecto tratado durante este período de transición, relacionado directamente al proyecto de investigación, fue el diseño y adquisición de los laboratorios que darían soporte a este nuevo plan de estudios. Uno de ellos fue el Laboratorio del Data Center, concebido para dar soporte a las asignaturas del área de Infraestructura de TI, cuyo diseño e implementación se detallan en los posteriores acápites. 


\section{Requerimientos funcionales del data center académico}

Los requerimientos que se aplican a un DC convencional no serán utilizados en su totalidad en el DC académico propuesto, ya que su principal objetivo es servir de plataforma de soporte para el desarrollo de laboratorios para las asignaturas del área de Infraestructura de TI y no como plataforma de producción. Sin embargo, contará con características propias de los DC convencionales adecuados al ámbito académico, tales como disponer de un falso piso simulado, tableros de distribución, UPS redundantes, aire acondicionado especial, cableado eléctrico y de comunicaciones, etc., igual que con los equipos de hardware, almacenamiento y redes debidamente concentrados y ubicados dentro de un aula.

Con este esquema, los alumnos dispondrán de un acceso directo a los equipos del DC que les permitirá interactuar con ellos, y también de forma remota, ya sea desde las mesas de trabajo del mismo laboratorio, desde los otros dos laboratorios con los cuales estará interconectado, o utilizando comunicaciones inalámbricas WiFi desde el campus.

De igual forma, el alumno tendrá acceso a laboratorios virtuales (máquinas virtuales) denominados "laboratorios como servicios" (Laboratories as a Service LaaS) por medio de estaciones de trabajo, utilizando equipos tales como desktops, laptops, notebooks o tablets, ya que la capacidad de proceso y almacenamiento estará localizada en el DC configurado como nube privada.

Con el fin de identificar los requerimientos funcionales con mayor precisión, se construyó una matriz de correspondencia entre las diversas asignaturas del área de Infraestructura de TI y el ambiente de laboratorio que mejor podría soportar el dictado de clases y la realización de actividades académicas de corte práctico. Los laboratorios en referencia fueron el Laboratorio de Networking, el Laboratorio de Data Center y el Laboratorio de Mobile Devices. Además, se identificaron algunas asignaturas que podrían beneficiarse con el uso de todos los laboratorios citados, conforme se señala en la tabla 1, donde se resaltan, con color verde, las nuevas asignaturas del plan de estudios.

Posteriormente, se procedió a construir cuadros de requerimientos de los recursos de laboratorio que se necesitarían para impartir los contenidos conceptuales y prácticos de cada asignatura. Además, se identificaron como plenamente alineadas para la utilización del Laboratorio de DC a las siguientes asignaturas:
a) Arquitectura de Computadoras
b) Sistemas Operativos
c) Taller de Sistemas Operativos
d) Arquitectura del Data Center 
Tabla 1

Asignaturas beneficiadas con la adecuación de los laboratorios

\begin{tabular}{|l|c|l|l|}
\hline Asignatura & Nivel & Carácter & Laboratorio \\
\hline Arquitectura de Computadoras & 3 & Obligatorio & Data Center \\
\hline Sistemas Operativos & 4 & Obligatorio & Data Center \\
\hline Comunicación de Datos & 5 & Obligatorio & Networking \\
\hline Taller de Sistemas Operativos & 5 & Obligatorio & Data Center \\
\hline Sistemas Operativos para Dispositivos Móviles & 6 & Electivo & Movile Device \\
\hline Redes de Computadoras & 6 & Obligatorio & Networking \\
\hline Seminario de Conectividad CCNA & 6 & Electivo & Networking \\
\hline Redes Inalámbricas & 7 & Obligatorio & Movile Device \\
\hline Seguridad de Redes & 7 & Obligatorio & Networking \\
\hline Seminario de Redes CCNA & 7 & Electivo & Networking \\
\hline Planeamiento de Redes Telemáticas & 8 & Obligatorio & Todos \\
\hline Arquitectura del Data Center & 8 & Obligatorio & Data Center \\
\hline Nuevas Tecnologías de la Información & 8 & Electivo & Todos \\
\hline Gestión de Servicios de TI & 10 & Obligatorio & Todos \\
\hline Gestión de TI & 9 Obligatorio & Todos \\
\hline Seguridad de la Información & Todos \\
\hline
\end{tabular}

\section{Elaboración propia}

Las tablas 2, 3, 4 y 5 muestran la relación del objetivo general y los principales objetivos específicos de las asignaturas indicadas, con los recursos de laboratorio requeridos para el dictado real y efectivo de los temas conceptuales y prácticos que permitan alcanzar estos objetivos. Igualmente, se presenta el nivel de disponibilidad de los recursos de laboratorio antes del inicio del proyecto, los cuales podrían ser los siguientes: 
a) Mínimo: implica que el recurso existe o está disponible en un nivel muy básico, posiblemente mediante simulación o virtualización primaria, desplegada en nivel limitado en una computadora personal de escritorio.

b) No disponible: hace referencia a que no existe el recurso de laboratorio requerido, que claramente señala la imposibilidad de abordar de forma coherente y efectiva el dictado de los tópicos en cuestión.

Tabla 2

Recursos requeridos de la asignatura Arquitectura de Computadoras

\begin{tabular}{|c|c|c|}
\hline \multicolumn{3}{|c|}{ Arquitectura de Computadoras } \\
\hline Objetivo & Recurso de laboratorio requerido & Estado \\
\hline $\begin{array}{l}\text { General } \\
\text { Identificar los componentes de } \\
\text { una computadora digital en ni- } \\
\text { vel de bloques }\end{array}$ & $\begin{array}{l}\text { Computadoras digitales de diver- } \\
\text { sos tipos: de escritorio, portátiles, } \\
\text { servidores de torre, servidores de } \\
\text { rack, servidores de chasis (blade) }\end{array}$ & \multirow{5}{*}{$\begin{array}{l}\text { Mínimo } \\
\text { Solo se dispone de com- } \\
\text { putadoras personales de } \\
\text { escritorio con las siguientes } \\
\text { características: } \\
\text { - Discos internos SATA. } \\
\text { - Solo una especificación } \\
\text { de ROM, RAM, Caché. } \\
\text { - Conjunto de interfaces } \\
\text { básicos de entrada/sali- } \\
\text { da. No se dispone de in- } \\
\text { terfaces clase "Enterprise" }\end{array}$} \\
\hline $\begin{array}{l}\text { computadoras } \\
\text { Determinar el rendimiento de la } \\
\text { computadora }\end{array}$ & $\begin{array}{l}\text { Sistemas de almacenamiento: disco } \\
\text { SATA, SAS, SSD, gabinetes de discos } \\
\text { externos, arreglos RAID }\end{array}$ & \\
\hline $\begin{array}{l}\text { Específico } \\
\text { Identificar y definir los elementos } \\
\text { básicos de una computadora }\end{array}$ & $\begin{array}{l}\text { Computadoras digitales de diver- } \\
\text { sos tipos: de escritorio, portátiles, } \\
\text { servidores de torre, servidores de } \\
\text { rack, servidores de chasis (blade) }\end{array}$ & \\
\hline $\begin{array}{l}\text { Específico } \\
\text { Identificar y comprender los princi- } \\
\text { pios de jerarquía de memoria }\end{array}$ & $\begin{array}{l}\text { Computadoras digitales con diver- } \\
\text { sos tipos y cantidades de memoria: } \\
\text { ROM, RAM, Caché }\end{array}$ & \\
\hline $\begin{array}{l}\text { Específico } \\
\text { Explicar el funcionamiento de las } \\
\text { interfaces de entrada/salida }\end{array}$ & $\begin{array}{l}\text { Computadoras digitales con diver- } \\
\text { sos tipos y cantidades de interfaces } \\
\text { de entrada/salida }\end{array}$ & \\
\hline
\end{tabular}

\section{Elaboración propia}


Tabla 3

Recursos requeridos de la asignatura Sistemas Operativos



Elaboración propia 
Tabla 4

Recursos requeridos para la asignatura Taller de Sistemas Operativos

\begin{tabular}{|c|c|c|}
\hline \multicolumn{3}{|c|}{ Taller de Sistemas Operativos } \\
\hline Objetivo & $\begin{array}{l}\text { Recurso de laboratorio } \\
\text { requerido }\end{array}$ & Estado \\
\hline $\begin{array}{l}\text { General } \\
\text { Implementar y configurar una } \\
\text { interacción eficiente entre clien- } \\
\text { tes y servidores } \\
\text { Administrar usuarios, servicios y } \\
\text { aplicaciones escalables, fiables y } \\
\text { seguros }\end{array}$ & $\begin{array}{l}\text { Computadoras con distintas po- } \\
\text { tencialidades y áreas de aplicación }\end{array}$ & \multirow{4}{*}{$\begin{array}{l}\text { Mínimo } \\
\text { Solo se dispone de com- } \\
\text { putadoras personales de } \\
\text { escritorio } \\
\text { Se emplea servidores } \\
\text { virtualizados } \\
\text { No se emplea la conec- } \\
\text { tividad LAN existente } \\
\text { (Interacción PC - MV) }\end{array}$} \\
\hline $\begin{array}{l}\text { Específico } \\
\text { Adquirir conocimientos de los } \\
\text { principios, fundamentos de fun- } \\
\text { cionamiento e implementación } \\
\text { de servicios de red }\end{array}$ & $\begin{array}{l}\text { Computadoras con distintas po- } \\
\text { tencialidades y áreas de aplicación } \\
\text { interfaces de red, medios y disposi- } \\
\text { tivos de conectividad }\end{array}$ & \\
\hline $\begin{array}{l}\text { Específico } \\
\text { Identificar procesos, políticas y } \\
\text { mecanismos para la administra- } \\
\text { ción de servicios de red imple- } \\
\text { mentados }\end{array}$ & $\begin{array}{l}\text { Computadoras con distintas po- } \\
\text { tencialidades y áreas de aplicación } \\
\text { donde experimentar la instalación, } \\
\text { configuración y operación de ser- } \\
\text { vicios de red }\end{array}$ & \\
\hline $\begin{array}{l}\text { Específico } \\
\text { Experimentar la instalación, con- } \\
\text { figuración y operación de los SO } \\
\text { Linux y Windows en el nivel de } \\
\text { servicios de red }\end{array}$ & $\begin{array}{l}\text { Computadoras con distintas } \\
\text { potencialidades y áreas de aplica- } \\
\text { ción donde experimentar la insta- } \\
\text { lación, configuración y operación } \\
\text { de servicios de red } \\
\text { Interfaces de red, medios y disposi- } \\
\text { tivos de conectividad }\end{array}$ & \\
\hline
\end{tabular}

\section{Elaboración propia}


Tabla 5

Recursos requeridos de la asignatura Arquitectura del Data Center

\begin{tabular}{|c|c|c|}
\hline \multicolumn{3}{|c|}{ Arquitectura del Data Center } \\
\hline Objetivo & Recurso de laboratorio requerido & Estado \\
\hline $\begin{array}{l}\text { General } \\
\text { Estudio de las principales arqui- } \\
\text { tecturas de servidores y alma- } \\
\text { cenamiento }\end{array}$ & $\begin{array}{l}\text { Servidores con distintas potencialidades y áreas } \\
\text { de aplicación } \\
\text { Sistemas de almacenamiento (diversas tecnolo- } \\
\text { gías e interfaces de disco clase Enterprise, a nivel } \\
\text { local y remoto) }\end{array}$ & \\
\hline $\begin{array}{l}\text { de infraestructura } \mathrm{TI} \text { en la or- } \\
\text { ganización }\end{array}$ & $\begin{array}{l}\text { Infraestructura de TI que permita implementar, } \\
\text { monitorear y analizar servicios de infraestructura } \\
\text { de TI (HPC, almacenamiento, virtualización, redes } \\
\text { convergentes, etc.) }\end{array}$ & \\
\hline $\begin{array}{l}\text { Específico } \\
\text { Diseño y estructura del DC } \\
\text { Consolidación, energía y HVAC }\end{array}$ & $\begin{array}{l}\text { Infraestructura de TI que permita explorar e identi- } \\
\text { ficar los componentes de infraestructura de un DC } \\
\text { Dispositivos e instalaciones anexas de genera- } \\
\text { ción, suministro y distribución de energía y HVAC }\end{array}$ & No disponible \\
\hline $\begin{array}{l}\text { Específico } \\
\text { Planeamiento, configuración e } \\
\text { implementación de servidores } \\
\text { y almacenamiento }\end{array}$ & $\begin{array}{l}\text { Servidores con distintas potencialidades y áreas } \\
\text { de aplicación } \\
\text { Sistemas de almacenamiento }\end{array}$ & \\
\hline $\begin{array}{l}\text { Específico } \\
\text { Implementación y prueba de } \\
\text { cluster, DR, virtualización, green } \\
\text { ITy cloud computing }\end{array}$ & $\begin{array}{l}\text { Servidores con distintas potencialidades y áreas } \\
\text { de aplicación } \\
\text { Sistemas de almacenamiento } \\
\text { Software de virtualización } \\
\text { Facilidades del tipo cloud computing en los niveles } \\
\text { de HW y SW que permitan el aprovisionamiento } \\
\text { elástico y bajo demanda de recursos virtualizados } \\
\text { de infraestructura de TI. }\end{array}$ & \\
\hline
\end{tabular}

Elaboración propia 
3. Requerimientos arquitecturales del data center académico

\subsection{Requerimientos generales}

Con base en las tendencias y estrategias de los cinco principales proveedores de DC de clase mundial (IBM, Dell, HP, Cisco y Oracle), y a reuniones efectuadas con los representantes locales de estos proveedores, se determinó que el diseño debía reflejar tanto la nueva tendencia de infraestructura unificada como el esquema tradicional. Es decir, con equipos de comunicación (switches) independientes para la red de servidores (switches LAN) y redes de almacenamiento (switches SAN). Esto último con fines académicos y para mostrar la evolución y las diferencias tecnológicas de estos enfoques. Por estas razones, el DC por adquirir debía cumplir con estas características fundamentales:

a) red interna unificada y tradicional

b) soporte de virtualización completa (servidores, almacenamiento y redes)

c) alta disponibilidad

d) modular y escalable

e) basado en estándares

f) software de gestión integrado con provisión de servicios tipo cloud computing

g) software de gestión de servicios basados en ITIL V3 2011

h) ambiente de DC acondicionado y adecuado para su operación dentro del aula (laboratorio del DC)

En la figura 2 se presenta un esquema global de los requisitos arquitecturales del data center académico.

Asimismo, se planteó que el data center académico (DCA) debía cumplir con las características que a continuación se detallan:

a) Arquitectura de servidores blade $\times 86$.

b) Arquitectura moderna de equipos de almacenamiento.

c) Diseño con alta disponibilidad parcial (redundancia en servidores, almacenamiento y UPS).

d) Capacidad suficiente para su uso de forma académica, y no como la de equipos de producción.

e) Configurado en una arquitectura virtualizada (data center virtualizado), utilizando el producto VMware, de la cual la Universidad ya cuenta con cierto nivel de conocimiento y experiencia.

f) Los equipos del DCA deben estar alojados en un ambiente simulado de uno real, siguiendo los lineamientos del estándar TIA-942 debidamente adaptados al ámbito académico, y con características cercanas a un "Tier 3" al que se denominará "Tier 3-Académico". 
g) Tableros de transferencia con llaves termomagnéticas. Uno de los tableros contará con tapas transparentes, para fines académicos, y los otros dispondrán indicadores luminosos de los diferentes puntos de interconexión.

h) El equipo de aire acondicionado será de tipo in row, recomendado para ambientes no productivos, sin redundancia, que simule la distribución de pasillo frío-pasillo caliente.

i) Utilización de cableado UTP categoría 6 para la conexión de las estaciones de trabajo del laboratorio; canalización suspendida al techo por medio de escalerillas tipo malla y canalización empotrada al piso, de modo que se puedan visualizar estos dos esquemas de canalización comunes en ambientes reales.

j) Equipos montados en gabinetes (racks) de tamaño estándar.

k) Tres pantallas permanentes LCD LED de 42" para el monitoreo de parámetros de operación y de una pizarra inteligente.

I) Interconexión del Laboratorio de DC con los laboratorios de networking y de mobile devices, ubicados en ambientes contiguos, utilizando enlaces redundantes de fibra óptica y de cobre.

\subsection{El Tier 3-Académico}

En el diseño del data center académico (DCA) se tomaron en cuenta los lineamientos de los estándares TIA 942 determinados por ADC Telecommunications (2006) y por el Uptime Institute LLC (2010), adaptados al ámbito académico en el que se utilizará.

\section{Software de gestión de nube}

\section{Software de gestión de servicios}

(basado en ITIL v3)

Virtualización

(Vmware)

Red interna

(unificada y tradicional)

Servidores blade $\mathbf{x} 86$

Storage externo (SAN)

Figura 2. Requisitos arquitecturales del data center académico Elaboración propia 
Es decir, como infraestructura de soporte al proceso de enseñanza-aprendizaje de las asignaturas del área de Infraestructura de TI. Como consecuencia de ello, las características del DC serán similares al Tier 3, por lo que se denominará "Tier 3-Académico"; estas características se resumen a continuación:

\section{A) Distribución de espacios}

a) El DCA será construido dentro de un aula, constituyéndose en el laboratorio del DC.

b) Estará ubicado en un espacio reservado, separado por lunas, del ambiente de trabajo de los alumnos, constituido por mesas de trabajo y estaciones (laptops).

c) Este espacio reservado al DCA es el que alojará la infraestructura tecnológica y contará con dos gabinetes de racks que alojarán los servidores (blade), unidades de almacenamiento, red interna de comunicaciones, UPS y equipo de aire acondicionado.

d) Contará con falso piso, tanto con fines académicos como para el despliegue del cableado de datos y de energía. La altura del falso piso no cumplirá con la recomendación del estándar y será pequeña, por no requerirse en un ambiente académico y por la poca altura del aula. Dispondrá de algunas tapas transparentes para poder observar el tendido del cableado.

e) Contiguo al espacio del DCA y separado por lunas corredizas existirá otro ambiente que alojará los tableros de distribución eléctrica, con un diagrama unifilar con señales luminosas en las tapas para fines académicos. Estos tableros estarán provistos de mecanismos de protección, ya que serán manipulados por los alumnos. Uno de ellos tendrá la tapa transparente con el fin de observar la parte interna, sin necesidad de abrirlo. La puerta metálica antifuego dará acceso al ambiente de trabajo de los alumnos.

\section{B) Infraestructura de cableado}

a) Se cumplirán las recomendaciones pertinentes del TIA 942 adaptado a su contexto.

b) El cableado de datos dentro de DCA se tenderá por el falso piso; al llegar a la zona de los alumnos se distribuirá por rejillas aéreas que atravesarán parte del laboratorio para descender por una columna y distribuirse por canaletas bajo el piso hasta las mesas de trabajo. El cableado aéreo se tenderá con fines exclusivamente académicos.

c) El cableado eléctrico en el DCA se instalará por el falso piso.

d) El cableado de datos será de tipo cobre (UTP 6) y fibra óptica (multimodo).

\section{C) Confiabilidad por capas}

El objetivo del DCA al cual está orientado no se adapta a la clasificación propuesta por los estándares, ya que su infraestructura no estará en fase 
productiva soportando aplicativos críticos de forma permanente. El DCA tampoco se mantendrá operativo 24 horas al día durante los siete días de la semana ( $24 \times 7$ ), ya que será apagado los fines de semana y en el período de vacaciones. Por consiguiente, es importante entender que su infraestructura no es de producción, sino de soporte a los laboratorios, o lo que podríamos denominar un esquema de laboratorios como servicios (LaaS).

\section{D) Frío, potencia y condiciones ambientales}

a) El DCA contará con un equipo especial de aire acondicionado (in row), ubicado entre los dos gabinetes, que generará condiciones ambientales similares a las de "pasillo frío" y "pasillo caliente". Cabe mencionar que el diseño adecuado de este equipo es un aspecto crítico, ya que de lo contrario se presentarían dificultades, tales como el hecho de que el aire caliente invada la zona de aire frío. Se considerará el uso de tapas ciegas dentro de los gabinetes y el encapsulamiento de la zona de aire caliente, si fuese necesario.

b) Los requerimientos de potencia fueron calculados con base en las necesidades de los equipos.

c) Para las consideraciones ambientales se consideraron los niveles de humedad, temperatura de operación y la arquitectura.

En la figura 3 se muestra un esquema simplificado de las principales características del Tier 3-Académico descrito en los párrafos anteriores.

Distribución de espacios

Laboratorio del data center - Aula de clases

Infraestructura de cableado

Eléctrico: falso piso

Datos (UTP 6 - fibra óptica): falso piso y rejillas

Confiabilidad por capas

Infraestructura académica - LaaS

Frío, potencia y condiciones ambientales

Aire acondicionado: Especial (in row)

Potencia: de acuerdo a las necesidades de los equipos

Figura 3. Tier 3-Académico

Elaboración propia 


\section{Principales actividades del proyecto de adquisición}

\subsection{Equipos y servicios adquiridos}

El proceso de selección, así como las negociaciones contractuales y la supervisión de la implementación del proyecto fueron realizadas por la Dirección de Informática y Sistemas (DIS) de la Universidad de Lima, que tuvo a su cargo la gestión del proyecto. A continuación se detallan los equiposy serviciosadquiridoseinstalados por la compañía IBM del Perú en relación con el laboratorio del DC y a la interconexión con los otros dos laboratorios.

A) Infraestructura:

a) posiciones hasta para 24 alumnos, dos operadores y un profesor

b) cableado de red integrado a la LAN privada del aula

c) tres pantallas LCD LED de 42"

d) una pizarra inteligente Mimio

B) Data center:
a) sistemas de frío
b) sistemas de potencia
c) sistemas de monitoreo

C) Configuración de hardware y software:
a) servidores
b) storage
c) comunicaciones
d) software de aplicación

D) Gabinetes (racks)

a) distribución de dos racks de medidas estándar (42u) en disposición central y longitudinal para creación de "pasillo frío - pasillo caliente"

\section{E) Sistemas de frío}
a) distribución "pasillo frío - pasillo caliente"
b) aire acondicionado in row por ser de fácil mantenimiento y recomendado para disposiciones de racks contiguos
c) sistema recomendado para ambientes no productivos
d) despliegue visual de componentes para mejor comprensión

\section{F) Sistemas de potencia}

a) simulación de doble alimentación y características equivalentes a Tier 3

b) doble acometida de alimentación pública

c) tableros de transferencia con llaves termomagnéticas 
d) doble UPS en configuración paralela

e) despliegue visual (planchas transparentes) para mejor comprensión

\section{G) Sistema de aire acondicionado}

a) implementación de dos (2) puntos de aire acondicionado para el centro de procesamiento: uno (1) para el equipo que será provisto en esta etapa y otro para un crecimiento futuro

b) implementación de canalización y cableado para el equipo evaporador y condensador, realizados desde el tablero

\section{H) Sistema de tierra de comunicaciones}

Implementación de conductores y accesorios para el aterramiento de los equipos y gabinetes del Centro de Procesamiento, teniendo en cuenta los siguientes detalles:

a) aterramiento de los pedestales del falso piso así como de los gabinetes

b) suministro e instalación de una barra de tierra principal

c) conductores de calibres $35 \mathrm{~mm}^{2}, 16 \mathrm{~mm}^{2}$ y $6 \mathrm{~mm}^{2}$

\section{I) Sistemas de monitoreo}

Los sistemas de monitoreo propuestos incluyen:
a) Netbotz Appliance con capacidad de monitorear variables de operación: temperatura, humedad, aniego y humo
b) integración a alarmas sonoras y visuales
c) simulación de un sistema de extinción de incendios
d) Suite Tivoli de monitoreo de los elementos de hardware y software

\section{J) Servidores}

La configuración de los servidores blade es la siguiente:
a) dos (2) chasis BladeCenter ubicados en dos (2) gabinetes independientes
b) configuración redundante con doble alimentación
c) cada chasis cuenta con dos cuchillas HS22 de dos (2) núcleos cada una
d) soporte a sistemas operativos: Windows y Linux
e) soporte a hipervisores comerciales: ESXi (Vmware), XEN (Citrix)
f) Hyper-V (Microsoft), KVM (Red Hat)
g) soporte opcional a BladeCenter Open Fabric Manager (BOFM)
h) agentes de Tivoli para monitoreo remoto
i) IBM Systems Director para monitoreo de variables de los servidores

Por otra parte, se adquirió un (1) servidor de rack para soporte de administración y gestión: monitoreo, cloud, virtualización, storage y comunicaciones. En la figura 4 se muestran los gabinetes propuestos y los equipos incluidos: 

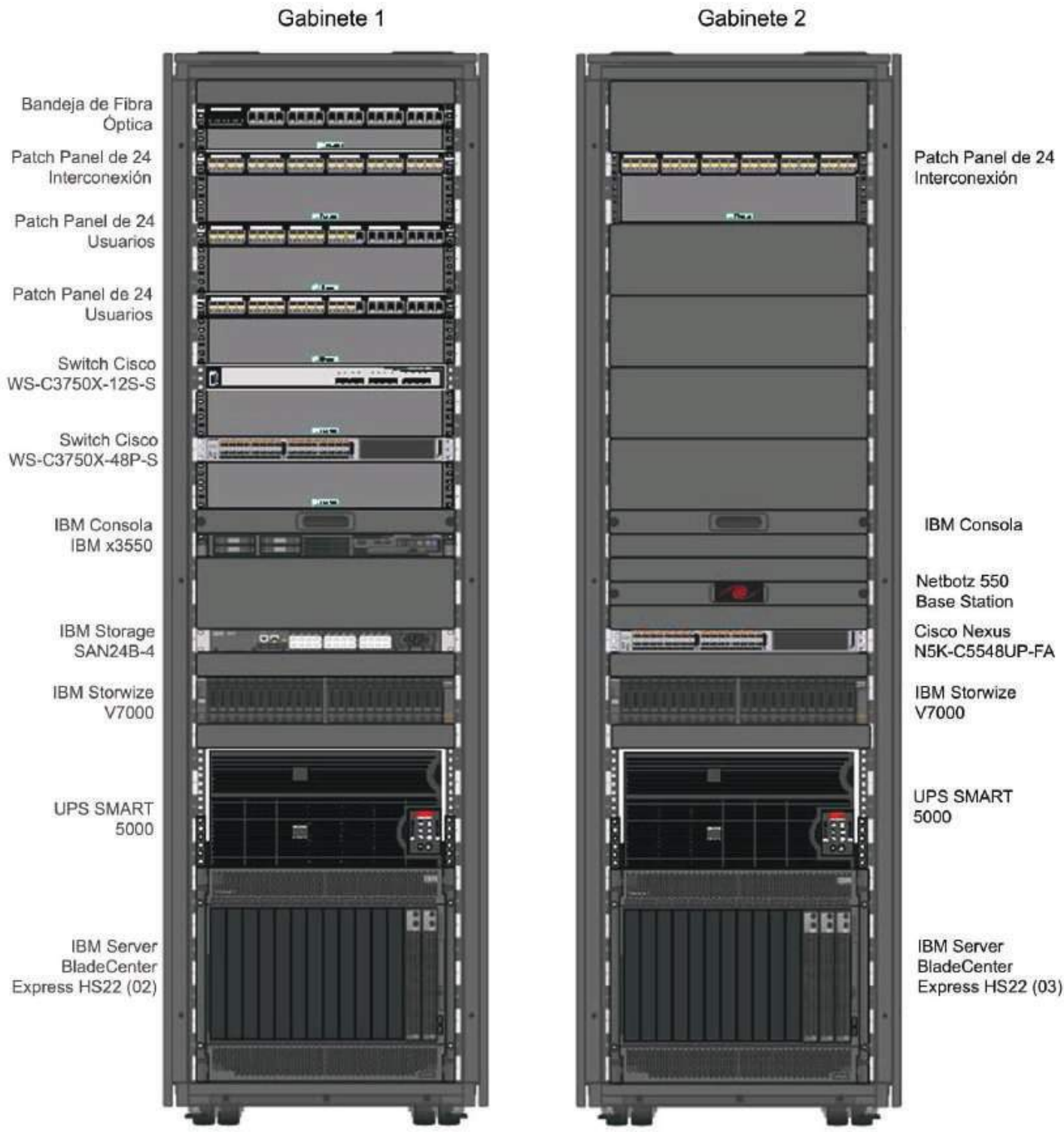

Figura 4. Gabinetes y equipos

Fuente: Carrera de Ingeniería de Sistemas - Universidad de Lima (2012) 


\section{k) Sistema de almacenamiento (storage)}

La configuración del almacenamiento es como se indica:
a) dos (2) equipos IBM Storwize v7000
b) siete (7) discos SAS 300GB 10K rpm por cada equipo
c) soporte a RAID0, RAID1, RAID5, RAID6, RAID10
d) discos con tecnología hot-swap
e) administración GUI
f) funciones de Thin-provisioning y Flash-copy
g) soporte opcional a réplicas Metro Mirror y Global Mirror
h) unidad externa de backup LTO
i) soporte a integración con Tivoli Storage Manager (TSM)

A continuación se exponen las principales características y funcionalidades del almacenamiento:
a) Thin-Provisioning: permite asignar una capacidad virtual de acuerdo con la demanda
b) Flash-copy: posibilita generar una copia instantánea de los discos para asig- narlos a otra partición
c) RAID: brinda seguridad lógica a los discos
d) LUN: permite asignar un disco lógico a cada partición

\section{L) Sistemas de comunicaciones}

Los equipos de comunicaciones incluyen:
a) dos (2) switches LAN con funcionalidades de capa 3
b) un (1) switch SAN
c) un (1) switch convergente Nexus 5000
d) dos (2) access points para la comunicación de los usuarios del aula
e) administración vía software de gestión incluid en los equipos
En la figura 5 se muestra el esquema de interconexión de los componentes del DC.

\section{M) Software de aplicación}

El software de aplicación cuenta con las siguientes características:
a) soportados por el Convenio de Iniciativa Académica firmado por la Universi- dad de Lima con IBM
b) suite de ISDM para provisionamiento y facturación en modalidad cloud 
c) suite de Tivoli para monitoreo

d) suite de Data Management para base de datos

e) suite de Websphere para motores transaccionales

f) suite de Rational para desarrollo y pruebas

N) Interconexión de laboratorios

En la figura 6 se muestra la interconexión de los laboratorios de Networking y de Mobile Devices con el laboratorio del DC.

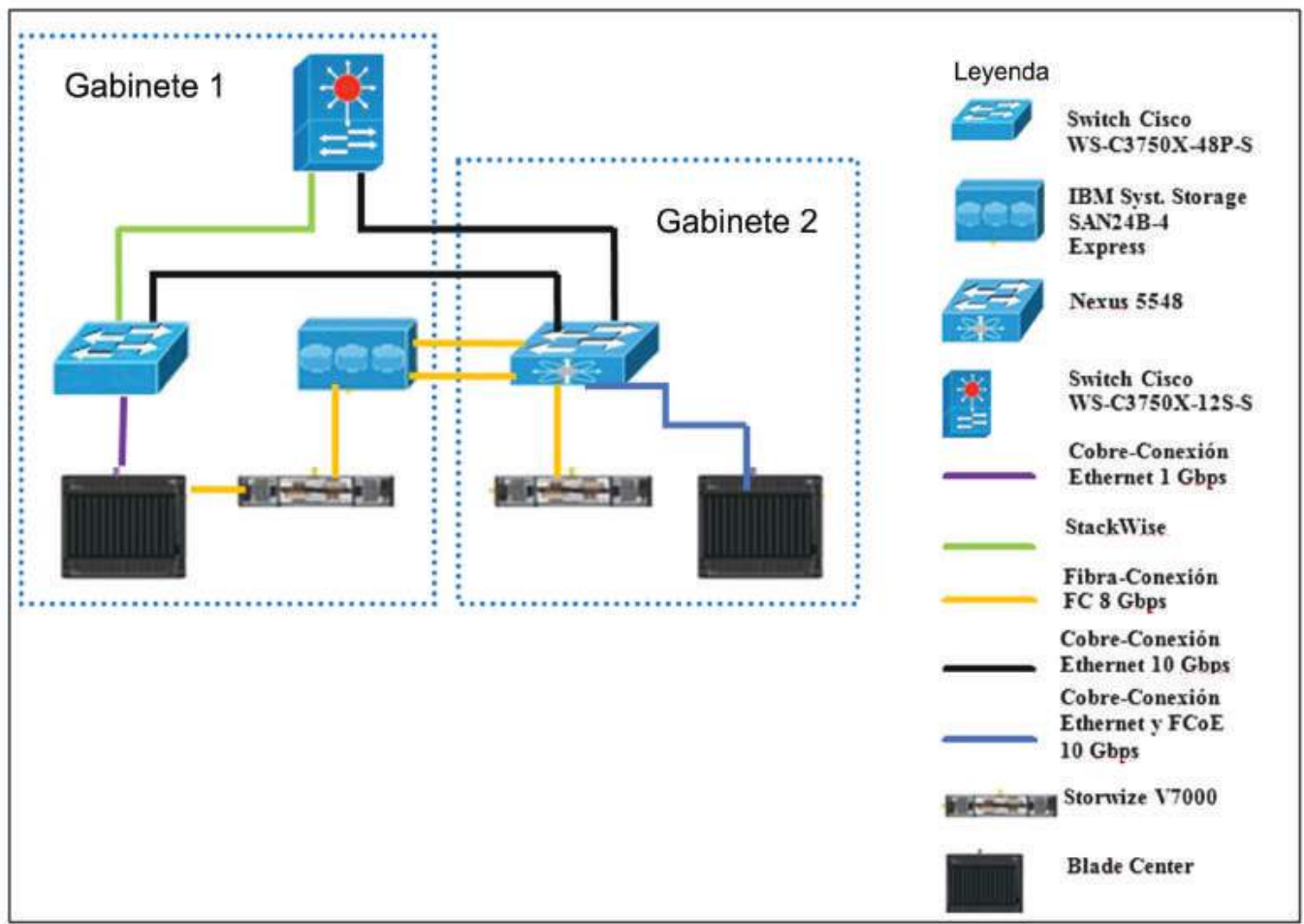

Figura 5. Red interna DCA

Fuente: Carrera de Ingeniería de Sistemas - Universidad de Lima (2012) 


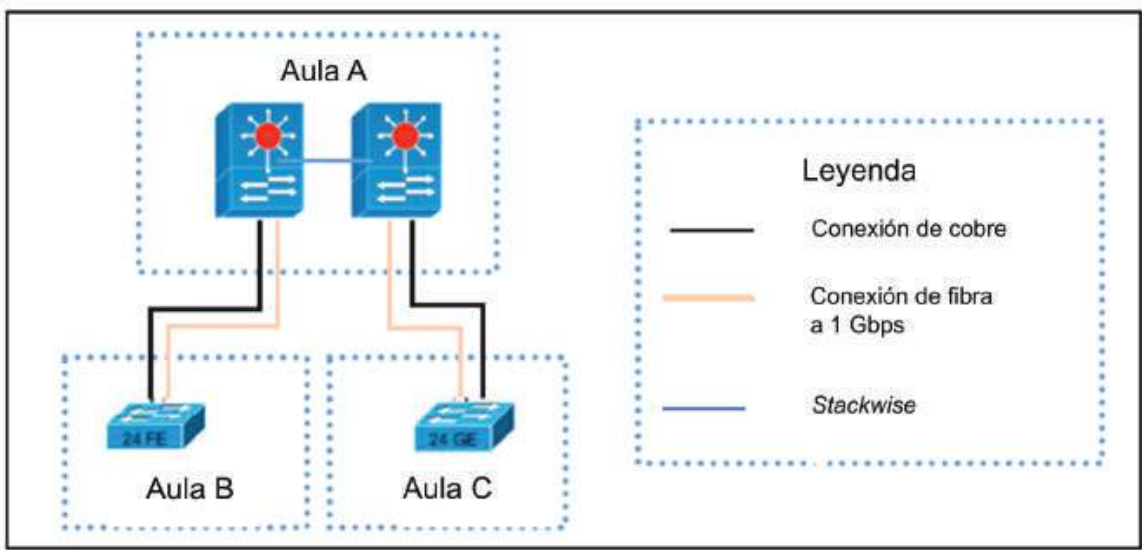

Figura 6. Interconexión de laboratorios

Fuente: Carrera de Ingeniería de Sistemas - Universidad de Lima (2012)

\subsection{Fotos del data center académico}

En la figura 7 se observan los dos ambientes del laboratorio. El área de trabajo de los alumnos, constituida por seis mesas de trabajo, cada una equipada con laptops en red utilizando cableado bajo piso y cableado aéreo. El ambiente del DC en el cual se aprecian los dos gabinetes separados por el equipo de aire acondicionado y en la parte superior se observan los ductos de extracción de aire. Se puede apreciar también el mueble asignado al profesor, situado cerca a la pantalla LCD. Este mueble cuenta con una estación de trabajo conectada en red.

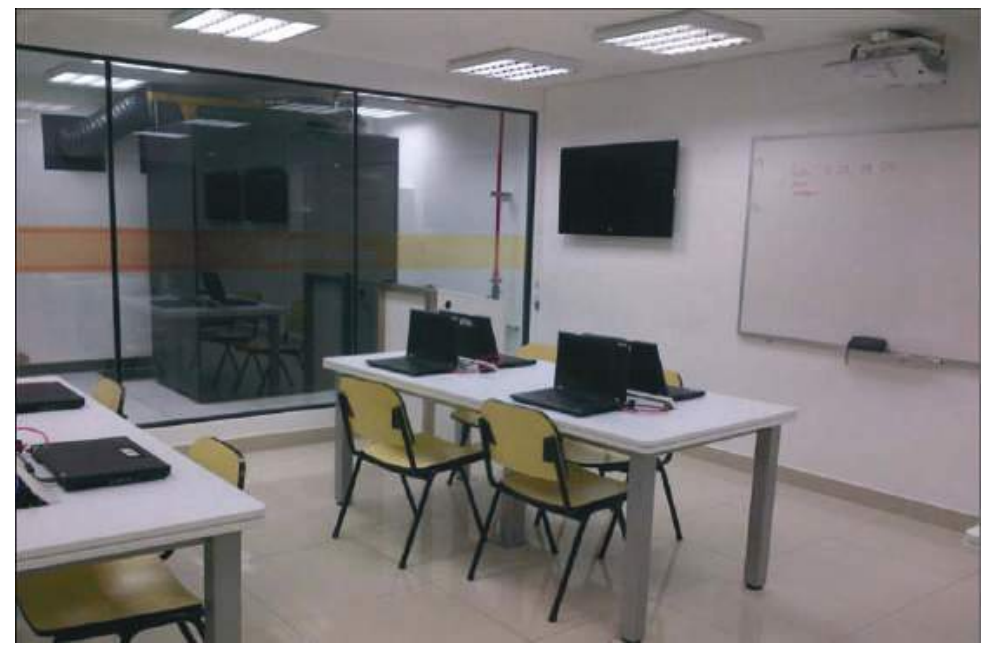

Figura 7. Mesas de trabajo en el DC

Fuente: Carrera de Ingeniería de Sistemas - Universidad de Lima (2012). 


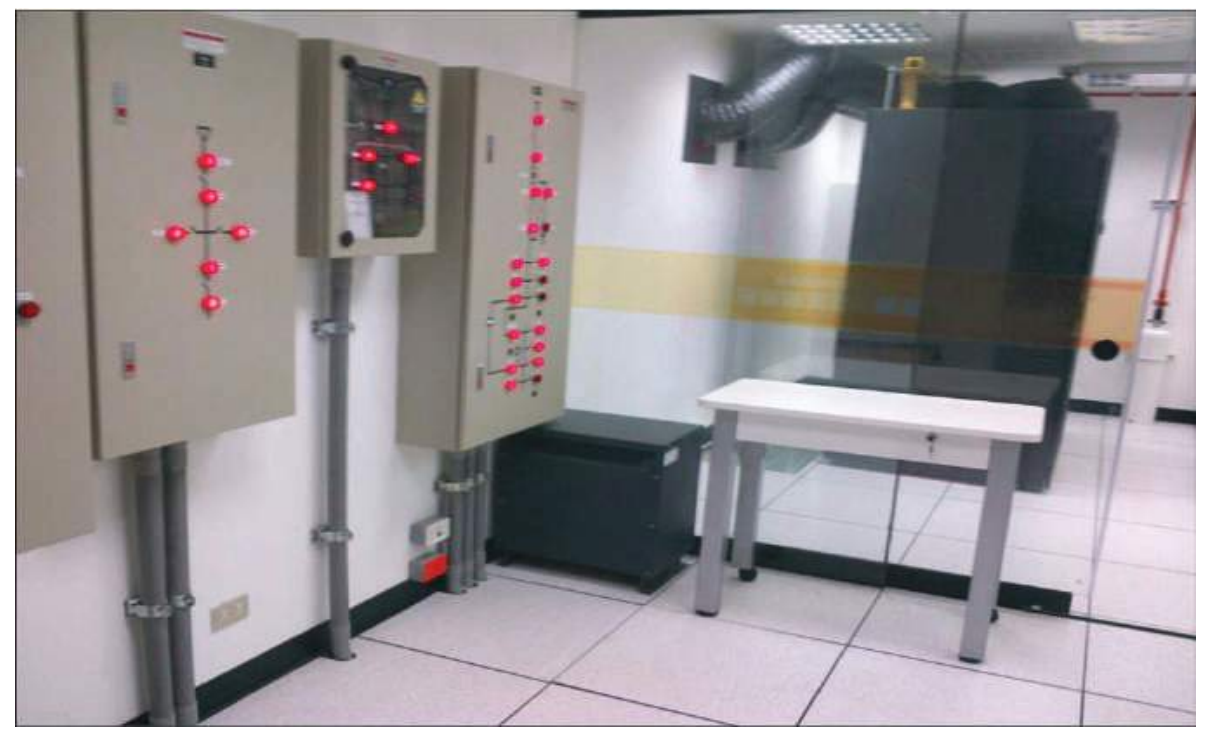

Figura 8. Tableros de distribución

Fuente: Carrera de Ingeniería de Sistemas - Universidad de Lima (2012)

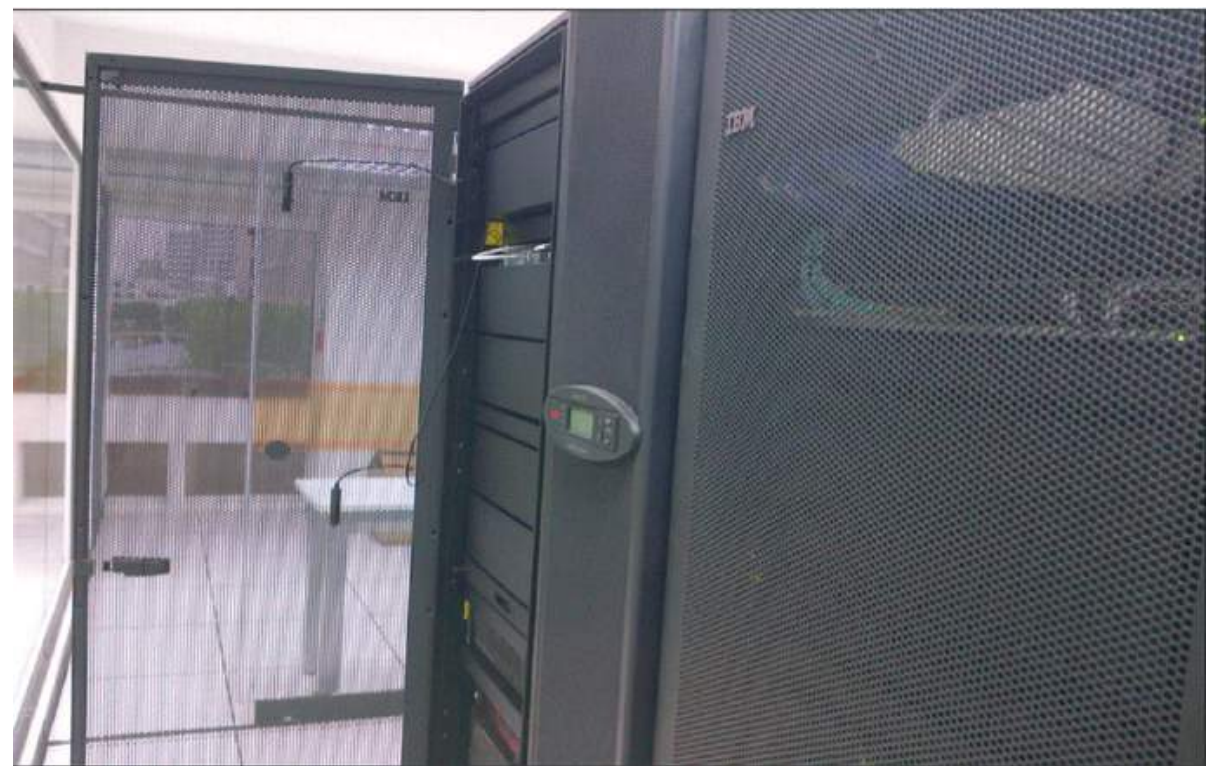

Figura 9. Gabinetes de equipos en el DC

Fuente: Carrera de Ingeniería de Sistemas - Universidad de Lima (2012) 
En la figura 8 se muestra el cuarto de los tableros de distribución, un tablero pequeño con tapa transparente; los otros presentan su esquema de distribución en la tapa resaltada con luces. Al ser abiertas estas tapas muestran en su interior una lámina de protección de material aislante de color transparente. Los tableros son los que reciben la energía comercial y la distribuyen a los diferentes ambientes, de acuerdo a sus necesidades y características particulares. En la parte baja de uno de los tableros y junto a la mesa de trabajo se observa un equipo transformador. Al fondo se aprecia el ambiente del DC, un balón (simulado) de gas FM-200 para extinción de incendios; y el falso piso de estos ambientes.

En la figura 9 se observan los dos gabinetes de equipos, separados por el equipo de aire acondicionado, ubicados en el ambiente del DC, en los cuales se alojan los diversos equipos, tales como los servidores (blade y rack), el almacenamiento, los dispositivos de red, el cableado interno, los UPS, la unidad de cinta y los equipos de monitoreo de condiciones ambientales.

En la figura 10 se observan las tapas transparentes del falso piso que permiten visualizar el cableado de transmisión de datos, así como el cableado eléctrico que pasa por debajo. Para una mejor visualización estos ambientes están iluminados.

En la figura 11 se observa el cableado aéreo, y en la figura 12 se dispone de una vista amplia desde el cuarto de tableros de distribución, en la que se observa el ambiente del DC y el de los alumnos con sus mesas de trabajo. Se aprecia también una de las tres pantallas LCD y la pizarra inteligente con su respectivo proyector.

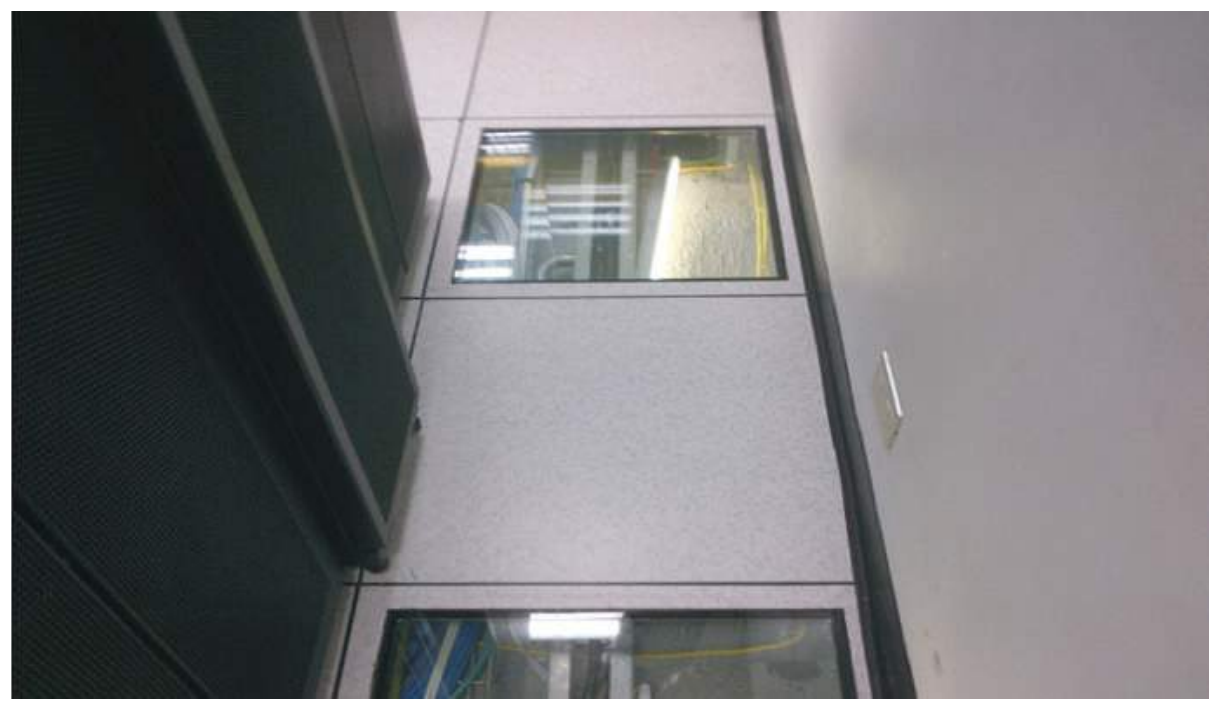

Figura 10. Tapas del falso piso transparentes Fuente: Carrera de Ingeniería de Sistemas - Universidad de Lima (2012) 


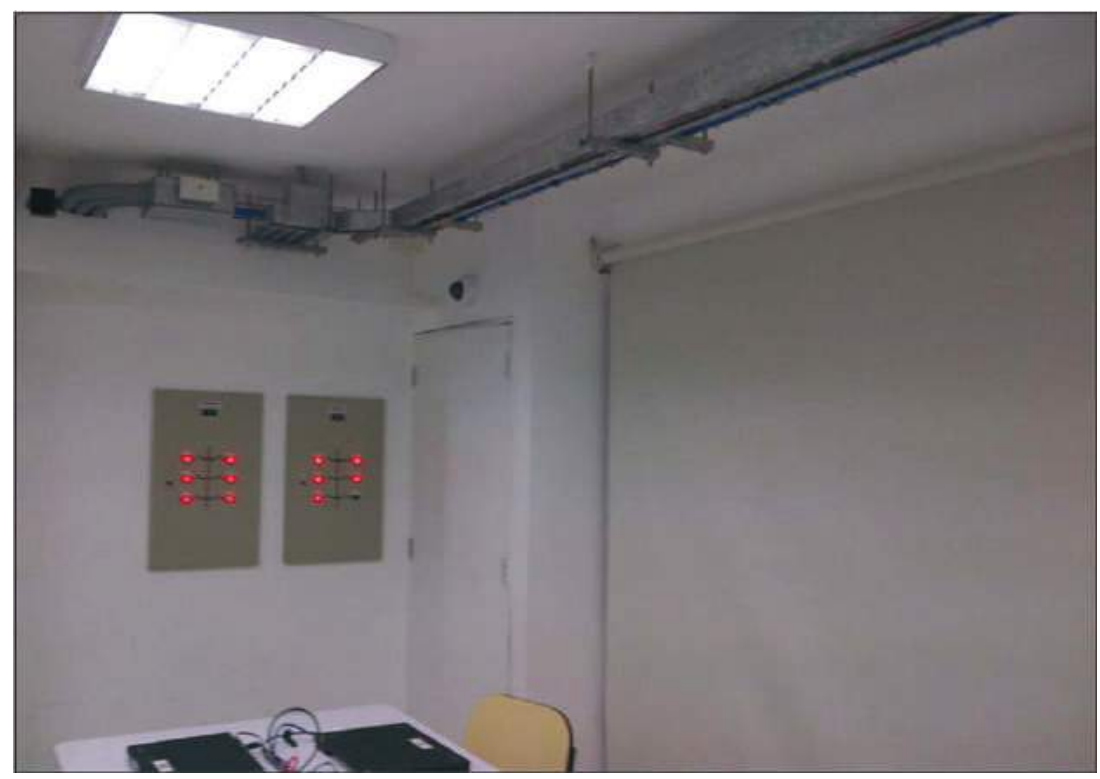

Figura 11. Cableado aéreo en área de alumnos Fuente: Carrera de Ingeniería de Sistemas - Universidad de Lima (2012).



Figura 12. Data center con vista a mesas de trabajo Fuente: Carrera de Ingeniería de Sistemas - Universidad de Lima (2012) 


\section{Utilización del laboratorio de data center}

\subsection{Utilización del laboratorio en las asignaturas del nuevo plan de estudios}

En este punto cabe señalar que si bien el laboratorio de DC es un compendio de diversas tecnologías de clase empresarial (servidores Blade, almacenamiento virtualizado Storwize, redes convergentes LAN-SAN, entre otros) su infraestructura puede ser empleada de forma básica o de formas muy elaboradas, dependiendo de los conceptos que el docente desee mostrar y validar. Las ventajas de emplear el laboratorio van desde la posibilidad de asignar al alumno infraestructura virtual personalizada, en el sentido de que cada alumno opera su propio ambiente de pruebas; hasta el acceso y empleo de tecnologías de computación avanzadas que usualmente solo se encuentran en ambientes de cómputo empresarial de gama media-alta, donde por hallarse en producción la experimentación o las pruebas están prohibidas o limitadas.

Desde ese punto de vista, cualquier asignatura que emplee infraestructura de cómputo se vería beneficiada del empleo del laboratorio, y si bien el colectivo de profesores del área de Infraestructura de TI de la carrera incluyó alguna(s) actividad(es) práctica(s) en el laboratorio de DC como parte del dictado de clases, se podría afirmar que la asignatura que más ha utilizado el laboratorio durante el primer período académico desde la puesta en servicio ha sido Arquitectura del Data Center, habiéndose programado siete (7) actividades de laboratorio para todo el período, las que se muestran en la tabla 6.

Tabla 6

Talleres realizados por la asignatura Arquitectura del Data Center - Período académico 2012-2

\begin{tabular}{|c|l|}
\hline Taller & \multicolumn{1}{|c|}{ Actividad } \\
\hline 1 & $\begin{array}{l}\text { Dimensionamiento de HVAC y energía } \\
\text { (HP Power Calculator XLS) }\end{array}$ \\
\hline 2 & $\begin{array}{l}\text { Diseño modular de racks y puntos de distribución (POD) } \\
\text { (HP Power Advisor y diagramas de bloques) }\end{array}$ \\
\hline 3 & Monitoreo de variables ambientales y de energía del data center (Netbotz, PDU) \\
\hline 4 & $\begin{array}{l}\text { Implementación y prueba de plataforma de virtualización ESX } \\
\text { (empleo de ESX 4.1, Blade Chassis y Storwize V7000) }\end{array}$ \\
\hline 5 & $\begin{array}{l}\text { Implementación y prueba de RAID 1 y 5 } \\
\text { (sobre Linux, CentOS v. 5.6) }\end{array}$ \\
\hline 7 & $\begin{array}{l}\text { Implementación y prueba de gestión lógica de volúmenes, LVM } \\
\text { (sobre Linux, CentOS v. 5.6) }\end{array}$ \\
\hline 7 & $\begin{array}{l}\text { Implementación y prueba de balanceo de carga por red, NLB } \\
\text { (sobre Windows Server 2003) }\end{array}$ \\
\hline
\end{tabular}

\section{Elaboración propia}


Del conjunto de talleres detallados, los dos primeros están enfocados al reconocimiento, exploración y determinación de especificaciones y capacidades de la infraestructura, mientras que los cinco restantes están orientados al empleo de las distintas capacidades del laboratorio para mostrar, verificar y consolidar los conceptos impartidos en clase.

\subsection{Procedimiento para la realización de una sesión en el laboratorio}

Tal como se señaló, las actividades académicas de corte práctico en el laboratorio de DC no diferirían de aquellas realizadas en otro laboratorio con facilidades de provisión de infraestructura de cómputo, básicamente capacidad de virtualización e idealmente posibilidad de reconfiguración del escenario de pruebas bajo demanda. En la tabla 7 se muestra la secuencia de pasos que implica la realización de una actividad práctica en el laboratorio de DC.

A modo de ejemplo, se revisará con mayor detalle cada una de las actividades (pasos) realizadas en los talleres efectuados en la asignatura Arquitectura del Data Center. Para el efecto, se tomará como ejemplo el taller N. 6: "Implementación y prueba de gestión lógica de volúmenes, LVM".

\subsubsection{Definición del alcance de la actividad}

Independientemente de si la actividad se realiza en el laboratorio de DC o en otra aula, el docente deberá determinar qué tópicos del contenido curricular ameritan demostración y verificación práctica. El punto clave de este paso de la secuencia es que establece el escenario de pruebas sobre el cual trabajará el alumno, y de allí se pueden derivar los requerimientos de infraestructura virtual. La tabla 8 muestra el objetivo y los requerimientos para la realización del taller N. 6.

\subsubsection{Preparación del escenario 'virtual' de pruebas}

La preparación del escenario 'virtual' de pruebas consiste en asignar recursos de infraestructura física y convertirlos en virtuales para generar la infraestructura adecuada con la que se trabajará en la actividad práctica. Este aprovisionamiento de recursos 'inicial' permite crear un conjunto de elementos de infraestructura virtual 'modelo' que se agrupan y consolidan en una 'plantilla' (archivo de formato OVF o vApp), la cual sirve de insumo base para crear réplicas personalizadas para el trabajo de cada alumno. La figura 13 muestra la plantilla OVF correspondiente al taller en mención. 
Tabla 7

Pasos para la planificación y ejecución de una actividad práctica en el laboratorio de DC

\begin{tabular}{|c|c|c|c|}
\hline Paso & Actividad & Entregable & Responsable \\
\hline 1 & Definición del alcance de la actividad & $\begin{array}{l}\text { - Objetivos } \\
\text { - Requerimientos }\end{array}$ & Docente \\
\hline 2 & $\begin{array}{l}\text { Preparación del escenario de pruebas } \\
\text { "virtual" }\end{array}$ & $\begin{array}{l}\text { Generación de máquinas virtuales (MV), } \\
\text { plantillas (OVF) o dispositivos virtuales } \\
\text { (vApp, Appliances). Infraestructura vir- } \\
\text { tual "modelo" }\end{array}$ & Docente \\
\hline 3 & $\begin{array}{l}\text { Despliegue de la infraestructura vir- } \\
\text { tual "modelo" sobre la infraestructura } \\
\text { física del laboratorio de DC }\end{array}$ & $\begin{array}{l}\text { Despliegue de infraestructura virtual } \\
\text { "individual", para cada alumno }\end{array}$ & $\begin{array}{l}\text { Docente, } \\
\text { personal de } \\
\text { laboratorio, } \\
\text { alumnos }\end{array}$ \\
\hline 4 & $\begin{array}{l}\text { Realización de la actividad de labora- } \\
\text { torio. Desarrollo guiado }\end{array}$ & $\begin{array}{l}\text { Infraestructura virtual individual "con- } \\
\text { figurada" (cada alumno "construye" su } \\
\text { propio escenario) }\end{array}$ & $\begin{array}{l}\text { Docente, } \\
\text { alumnos }\end{array}$ \\
\hline 5 & Cierre de la actividad práctica & $\begin{array}{l}\text { Infraestructura virtual configurada } \\
\text { "suspendida" }\end{array}$ & $\begin{array}{l}\text { Docente, } \\
\text { alumnos }\end{array}$ \\
\hline 6 & $\begin{array}{l}\text { Revisión del nivel de avance y cumpli- } \\
\text { miento de la actividad (puede darse } \\
\text { durante la actividad o tras la finaliza- } \\
\text { ción de esta) }\end{array}$ & $\begin{array}{l}\text { Nota obtenida por el alumno en la } \\
\text { actividad }\end{array}$ & Docente \\
\hline 7 & $\begin{array}{l}\text { Descarte o preservación de la infraes- } \\
\text { tructura virtual trabajada (sea que ya } \\
\text { no se requiera o se trate de una activi- } \\
\text { dad continuada) }\end{array}$ & $\begin{array}{l}\text { Infraestructura física "liberada" y sin } \\
\text { asignación de recursos o grupo de } \\
\text { recursos preasignados (según sea des- } \\
\text { carte o preservación) }\end{array}$ & $\begin{array}{l}\text { Docente, per- } \\
\text { sonal de labo- } \\
\text { ratorio }\end{array}$ \\
\hline
\end{tabular}

Elaboración propia

Tabla 8

Objetivo y requerimientos del taller Implementación y prueba de LVM

\begin{tabular}{|l|l|}
\hline $\begin{array}{l}\text { Arquitectura del Data Center } \\
\text { Taller N. 6: Implementación y prueba de LVM }\end{array}$ \\
\hline \multirow{2}{*}{ Objetivos } & $\begin{array}{l}\text { - Implementar la funcionalidad Administración de volúmenes lógicos } \\
\text { (LVM) en servidores CentOS } \\
- \text { Comprobar el funcionamiento de dicho volumen } \\
- \text { Valorar sus ventajas comparativas con respecto a las soluciones basadas } \\
\text { en RAID }\end{array}$ \\
\hline Requerimientos & $\begin{array}{l}\text { - Servidor físico o ambiente virtualizado que permita el despliegue de ser- } \\
\text { vidores virtuales } \\
- \text { Plantilla OVF de Máquina Virtual (CentOS 5.6) } \\
- \text { Cliente FTP seguro (WinSCP) }\end{array}$ \\
\hline
\end{tabular}

Elaboración propia 


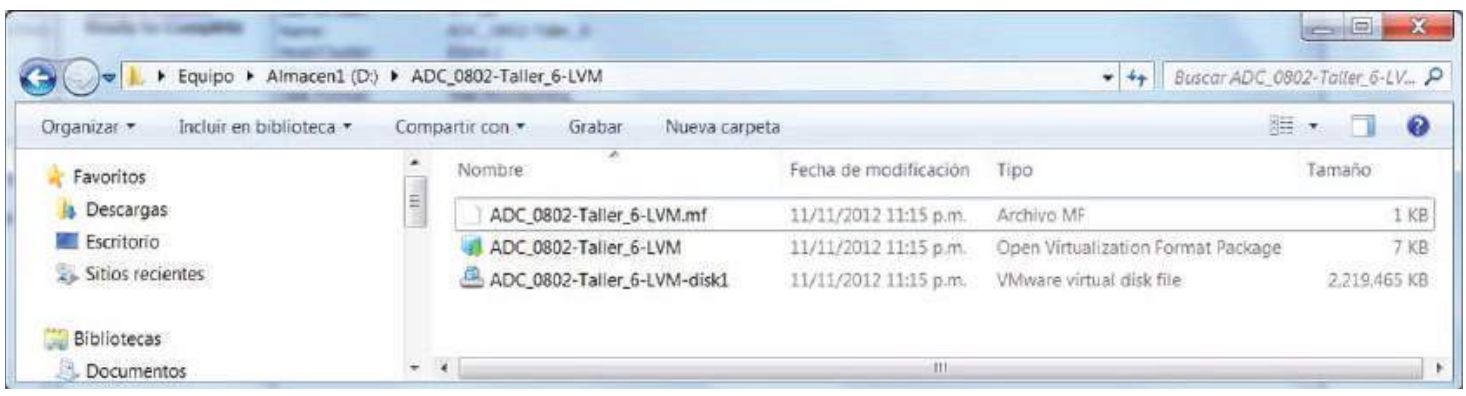

Figura 13. Plantilla OVF correspondiente al taller Implementación y prueba de LVM Elaboración propia

\subsubsection{Despliegue de la infraestructura virtual 'modelo' sobre la infraestructura física del laboratorio de data center}

En esta etapa se procede a crear copias de la infraestructura virtual 'modelo', llevándose a cabo alguna personalización ligera que relacione determinado despliegue con un alumno en particular. La posibilidad de que cada alumno cuente con acceso controlado a su infraestructura virtual garantiza que el avance



Figura 14. Despliegue de la plantilla OVF del taller de implementación y prueba de LVM Elaboración propia 
que logre no sea alterado por otro participante de la actividad práctica, pero a la vez le impone un mayor grado de responsabilidad para alcanzar los objetivos de la actividad. En la figura 14 se observa uno de los pasos del despliegue de la plantilla, y la figura 15 muestra una vista de los recursos físicos de almacenamiento que serán utilizados por cada alumno al trabajar con su infraestructura virtual.

En la figura 16 se observa una vista compuesta por paneles parciales tomados de cada una de las "cuchillas" (blade servers), donde se aprecian las instancias individuales de máquinas virtuales que cada alumno está trabajando.

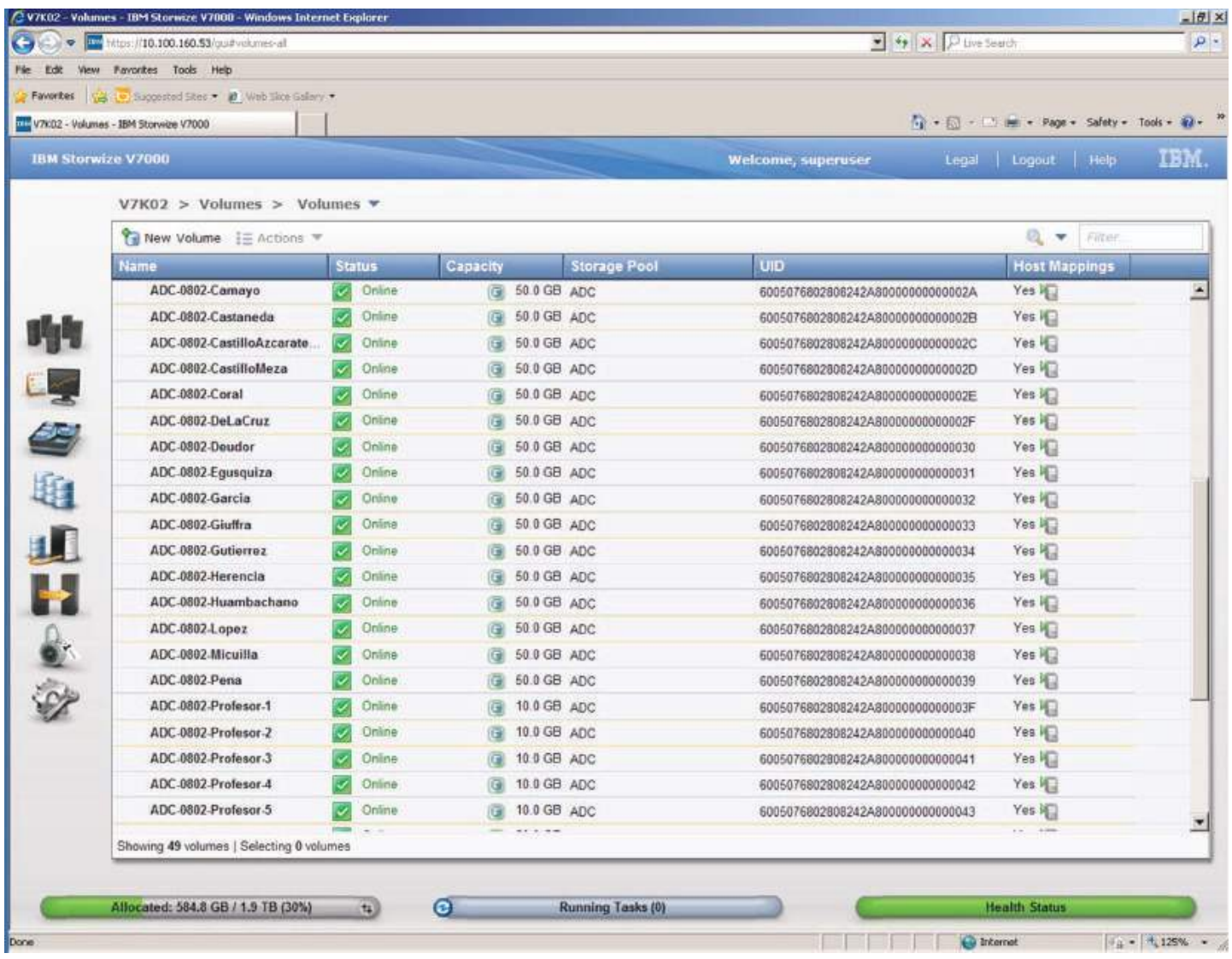

Figura 15. Recursos físicos de almacenamiento integrados a la infraestructura virtual Elaboración propia 


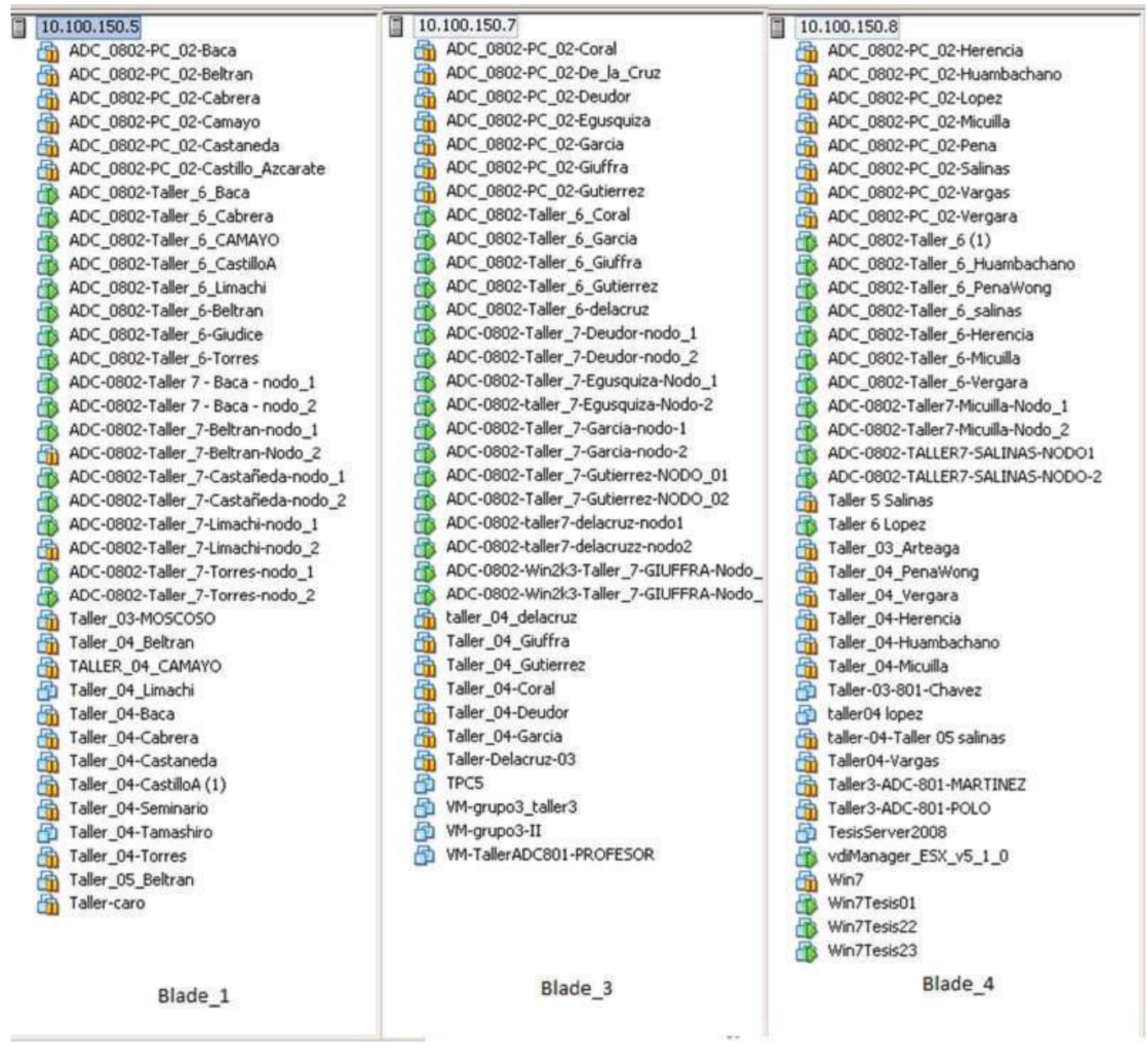

Figura 16. Conjunto de MV desplegadas para empleo de los alumnos Elaboración propia

\subsubsection{Realización de la actividad de laboratorio}

Una vez lista la infraestructura virtual se inicia la realización de la actividad académica práctica, donde los alumnos pueden trabajar directamente en el escenario y ganar de primera mano una valiosa experiencia con tecnologías clase Enterprise, que de otra forma no estarían disponibles. La figura 17 muestra una vista grupal de las pantallas empleadas como guía referencial para el desarrollo de la actividad, mientras que la figura 18 presenta una de estas vistas con mayor detalle. 


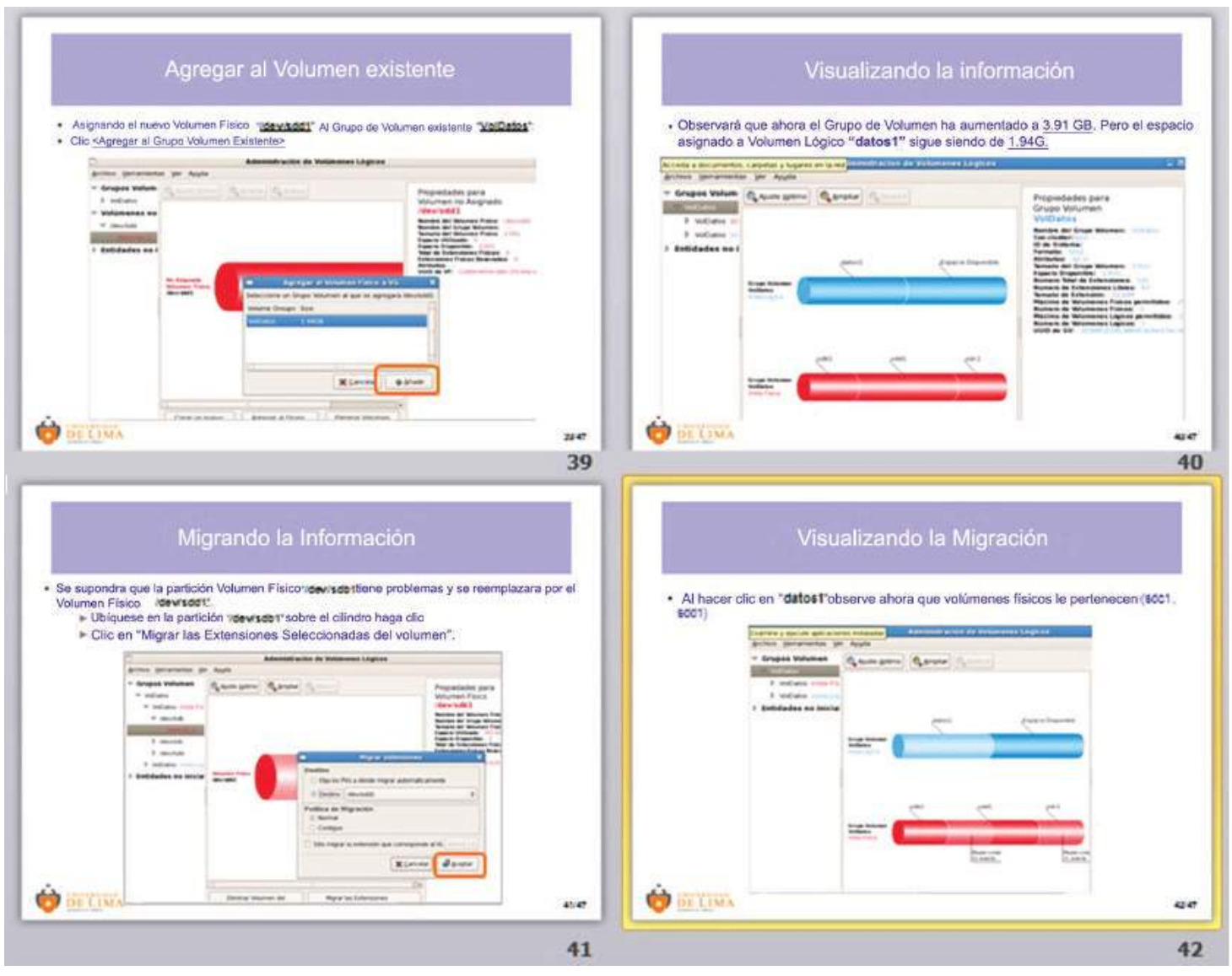

Figura 17. Vista grupal de la guía del taller Implementación y prueba de LVM Elaboración propia

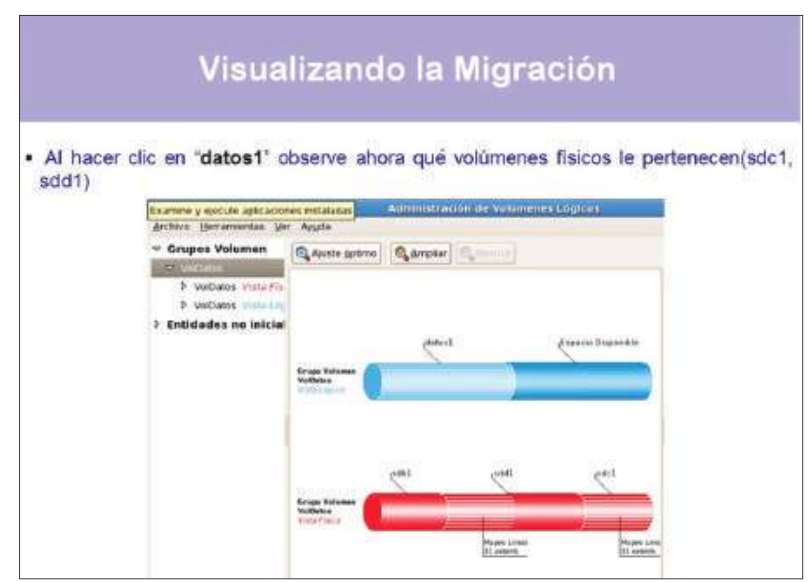

Figura 18. Vista en detalle de la guía referencial del taller Implementación y prueba de LVM Elaboración propia 


\subsubsection{Cierre de la actividad práctica}

Dependiendo de la actividad que se esté realizando, y siempre según el criterio del docente, el entregable de la sesión puede ser un reporte complementario o directamente 'la infraestructura' sobre la cual el alumno ha trabajado. Si se tratara de este último caso, el docente solicitará a los alumnos que "suspendan" las máquinas virtuales que forman parte del escenario en cierto momento o con cierta funcionalidad visible en la pantalla. Esto trae un doble beneficio, pues por simple inspección se puede verificar el cumplimiento de la disposición y asegurar el nivel de avance que presenta cada alumno. Además, facilitará la reactivación de las máquinas virtuales para la revisión en detalle y la evaluación por parte del docente. La figura 19 muestra una vista parcial con un conjunto de máquinas virtuales que se encuentran suspendidas (nótese la doble barra que simboliza dicho estado).
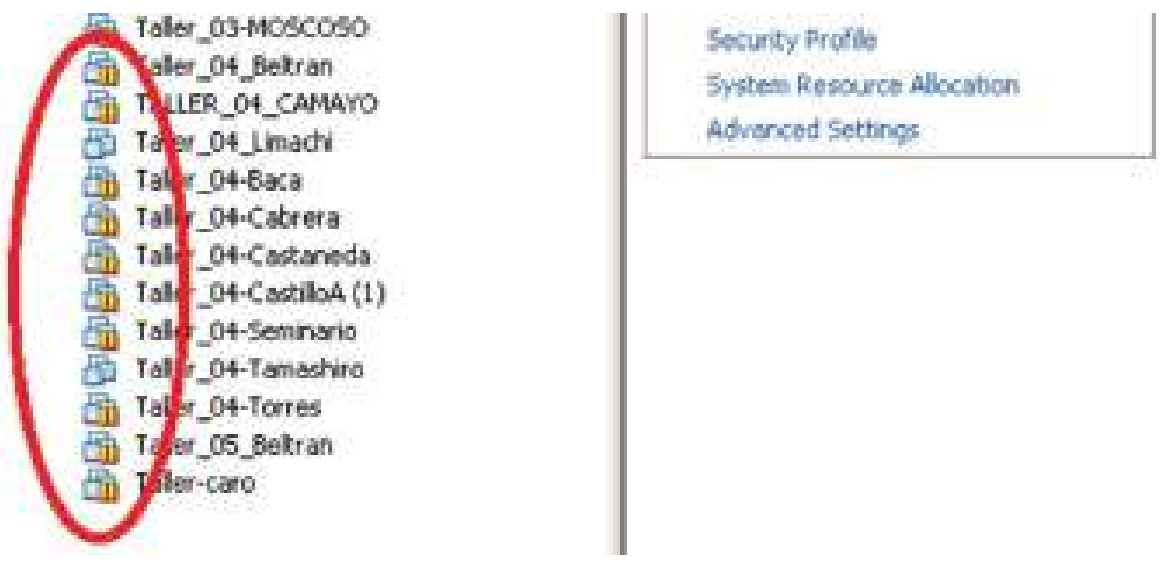

Figura 19. Vista parcial de un grupo de máquinas virtuales suspendidas Elaboración propia

\subsubsection{Descarte o preservación de la infraestructura virtual trabajada}

Aunque no es una regla, la gran mayoría de actividades prácticas desarrolladas en los laboratorios son actividades 'autocontenidas', donde el docente imparte los conceptos y pautas necesarios para la realización de la actividad. El trabajo realizado no es requisito o insumo de las posteriores actividades de laboratorio, salvo, posiblemente, en el plano cognitivo.

En este aspecto, el laboratorio de DC permite preservar temporalmente los despliegues realizados con el fin de que se continúe trabajando sobre ellos o se les complemente con actividades prácticas posteriores. Esto, a su vez, amplía el panorama de proyectos que se pueden abordar en una asignatura o 
en una secuencia de asignaturas, ya que podría tratarse de proyectos de mayor complejidad, los cuales requieran ser 'construidos' en varias sesiones de un mismo curso; o de mayor extensión, que trasciendan los límites de un curso y sean continuados en otra materia. Si este fuese el caso, la infraestructura virtual generada por cada alumno podría preservarse en los sistemas de almacenamiento del laboratorio de DC, en espera de ser reutilizada oportunamente. De no ser así, o tras haberse agotado la utilización de la infraestructura virtual, se procedería al descarte de dicha infraestructura, que en la práctica equivale a liberar los recursos que fueron asignados para tal fin.

La figura 20 muestra la situación del sistema de almacenamiento cuya capacidad nominal es de 2,1 terabytes, lo que nos deja una capacidad formateada disponible de aproximadamente 1,9 TB, pero que en la vista muestra una asignación 'virtual' de 2,5 TB, gracias al aprovisionamiento ligero Thin provisioning. Este último valor claramente supera la capacidad disponible, y si se usasen concurrentemente las infraestructuras virtuales aprovisionadas, los requerimientos desbordarían los recursos, generándose fallos en el nivel de acceso/provisión de recursos.



Figura 20. Sobreasignación de recursos virtuales en el nivel del sistema de almacenamiento Elaboración propia 
a) Se está consolidando la tendencia de construcción de los data center, como infraestructuras integradas, virtualizadas y gestionadas bajo el concepto de nube privada.

b) Las arquitecturas de los data center virtualizados empresariales pueden ser adaptados para su utilización en el ámbito académico universitario, como se mostró en el caso de estudio.

c) La Carrera de Ingeniería de Sistemas de todas las universidades deberían contar con un laboratorio de data center, que brinde soporte al proceso de enseñanzaaprendizaje de las asignaturas del área de Tecnologías de la Información.

d) Se están desarrollando e integrando marcos de trabajo (frameworks) que contemplan todos los aspectos relacionados con el diseño, la construcción y la operación de los data center, convirtiéndose en un área importante de estudio.

\section{Referencias}

ADC Krone (2006). TIA-942, Data Center Standards Overview. Recuperado de http:// www.herts.ac.uk/_data/assets/pdf_file/0017/45350/data-centre-standards.pdf

Universidad de Lima (2012). Ingeniería de sistemas - Perfil profesional. Recuperado de http://www.ulima.edu.pe/pregrado/ingenieria-de-sistemas/perfil-profesional

Uptime Institute, LLC (2010). Data Center Site Infrastructure. Tier Standard Topology. Recuperado de http://www.gpxglobal.net/wp-content/uploads/2012/08/ tierstandardtopology.pdf

Villarrubia, C. (2012). Cinco tendencias del data center en 2012, según Gartner. DatacenterDynamics. Recuperado de http://http://www.datacenterdynamics. es/focus/archive/2012/01/cinco-tendencias-del-data-center-en-2012seg\%C3\%BAn-gartner 